\title{
Traffic Matrix-Based Routing Optimization
}

\author{
Jia Ru \\ National Engineering Laboratory for Next Generation \\ Internet Interconnection Devices \\ School of Electronic and Information Engineering, Beijing \\ Jiaotong University \\ Beijing, China \\ 11111010@bjtu.edu.cn
}

Su Wei

National Engineering Laboratory for Next Generation Internet Interconnection Devices

\begin{abstract}
Smart Identifier NETwork (SINET) is a clean-slate future Internet architecture, which has the superiority of perceiving real-time traffic matrix. We use traffic matrix as the constraint, model on load balancing routing problem, and transform the primal problem into dual problem by using Lagrange function. Therefore, the optimal objection of the dual problem can be easily achieved. In this paper, we propose a traffic matrix based load balancing routing scheme to achieve the optimization goal of the dual problem. We use OMNET++ to run the experiment simulation and the result shows that, compared with traditional routing scheme, the traffic matrix based load balancing routing could reduce congestion effectively and realize load balancing.
\end{abstract}

Keywords-Smart Identifier NETwork (SINET); traffic matrix; routing optimization; Lagrange duality; load balance;

\section{INTRODUCTION}

In recent years, the future Internet architecture becomes the focus of research. Smart Identifier NETwork (SINET) is one of the "clean slate" designs of future Internet architecture, (former name Smart and Cooperative Networks / SCN) [1][4]. SINET is based on the basic framework and theories of three layers and two domains: the smart pervasive service layer (L-SPS), the dynamic resource adaption layer (L-DRA), the collaborative network component layer (L-CNC), the entity domain (D-EN) and the behavior domain (D-BE).

SINET has many advantages, such as information-centric, separation between controls and data, and adaptting reallocate network resource according to the fluctuation of environmental conditions. In this paper, we focuses on its ability of perceiving real-time traffic matrix, which performs between the L-DRA and the L-CNC.

Traffic matrix (TM) [5] reflect the volume of traffic that flows between all Origin-Destination pairs (OD) in a network. TM is a critical input to traffic engineering and transportation planning [6]. Troditional techniques [7] for estimating traffic matrix are based on some data acquisition methods like SNMP.

\author{
School of Electronic and Information Engineering, Beijing \\ Jiaotong University \\ Beijing, China \\ wsu@bjtu.edu.cn \\ Zhang Hongke \\ National Engineering Laboratory for Next Generation \\ Internet Interconnection Devices \\ School of Electronic and Information Engineering, Beijing \\ Jiaotong University \\ Beijing, China \\ hkzhang@bjtu.edu.cn
}

This kind of estimation has low accuracy and high overhead, and is hardly applied in large-scale networks. Since OpenFlow [8] makes it easier to record all active flows, the OpenTM [9] approach is propose to accurately estimate the traffic matrix in the Software-Defined Network (SDN). OpenTM has high accuracy but its controller has to bear the major overheads. SINET not only has the ability to record all active flows, but distribute the computational overhead into all Forwarding Nodes (FNs). In addiion, we have built a prototype [3] to compare the accuracy between SINET and OpenTM in different scenarios, and the results show that the traffic matrix estimation in SINET obviously outperforms that in OpenTM.

In this paper, taking advantages of the real-time traffic matrix in SINET, we first use the traffic matrix as the constraint, and model on load balancing routing problem. Secondly, we transform the primal problem into dual problem by using Lagrange function, since the optimization goal of the dual problem can be easily achieved. Thirdly, in order to achieve the dual problem optimization goal, we propose a traffic matrix based load balancing routing scheme. Lastly, we use OMNET++ to run the experiment simulation based on NSFnet topology. The results show that TMLB can better reduce congestion and realize load balancing, compared with traditional routing mechanism.

\section{THE TRAFFIC MATRIX ESTIMATION IN SINET}

The detail about SINET could be refer to our previous findings [1]-[4], this paper only covers some elements relevant to this article. There are three kinds of identifiers in the D-EN corresponding to three layers respectively: the Service Identifier (SID), the Family Identifier (FID) and the Node Identifier (NID). Note that Path Identifier (PID) is one kind of FID, which is used to identify the transmission resource for inter-domain routing.

Fig.7.1 illustrates the routing procedure in SINET. Domains D2 and D3 negotiate two paths P2 and P3. For a path that begins at a domain, the Resource Manager (RM) 
maintains the path's endpoint located at the domain and the domain identifier at which the other endpoint are located.

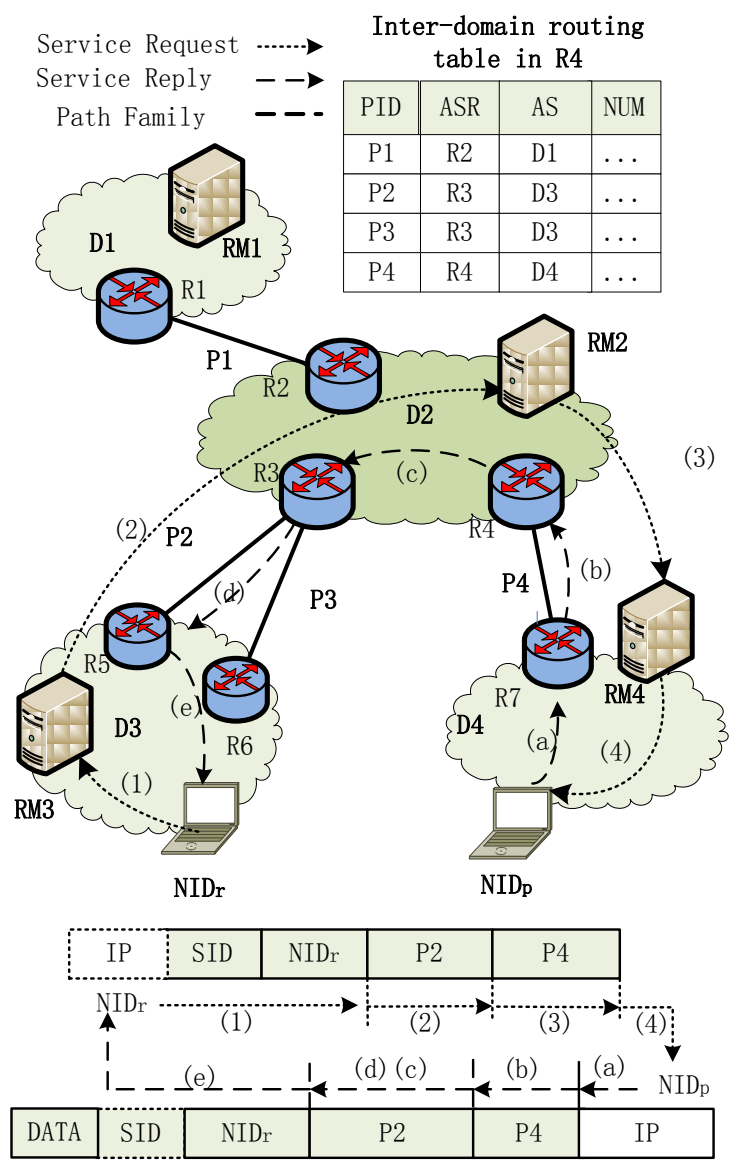

Fig.1 Routing procedure in SINET

Through the service registrstin mechanism, RM knows all reachability informations of all SIDs. Each border maintains an inter-domain routing table. When a service requester (identified by $\mathrm{NID}_{\mathrm{r}}$ ) wants to obtain a service represented by an SID, it sends out a service request message to its local RM. In Fig.7.1, the thin dotted arrow illustrates how a service request message is forwarded from a requester to a provider hosting the desired service data represented by an SID. When the provider $\left(\mathrm{NID}_{\mathrm{p}}\right)$ receives the service request message, it knows the desired SID, the requester's NID, and the interdomain paths to be used to reach the requester. The provider $\left(\mathrm{NID}_{\mathrm{p}}\right)$ encapsulates the desired data with a header that carries the requester's NID, the SID, and the PIDs, which is shown as message format (4). The bold dotted arrows in Fig.7.1 illustrates how the desired data is forwarded from a provider to a requester.

In SINET, the TM estimate is distributed into all border routers. To obtain the traffic matrix from an ASR to any other border routers in same domain, the border router only needs to count packets that contain the PID, and report its traffic matrices to the RM. the traffic matrices can be timely estimated in SINET, since a router is able to estimate the traffic matrix at line speed when it forwards data packets. In addition, the overhead of the traffic matrices estimation is acceptable [3].

\section{Mathematical Modeling}

There is a network topology $\boldsymbol{G}(\boldsymbol{V}, \boldsymbol{E}), \boldsymbol{V}$ is a set of all border routers in a domain, $|\boldsymbol{V}|=N$. $\boldsymbol{E}$ is a set of all links in a domain, $|\boldsymbol{E}|=L$. $\boldsymbol{T} \boldsymbol{M}$ is a matrix with $N$ rows and $N$ columns. The element in the $i$ row and $j$ column $(i \in \boldsymbol{V}, j \in \boldsymbol{V})$ donates the traffic demand from router $i$ to router $j$. If there are $M$ nonzero value in the matrix, $\boldsymbol{T M}=\{r 1, r 2, \cdots, r M$ (Mbps) $\mid r i j \neq 0$, $i \in \boldsymbol{V}, j \in \boldsymbol{V}\}$

\section{A. Load Balancing Routing Optimization}

The optimal object is to minimize the maximum link utilization $\theta$, Then the primal problem is:

$\min \theta$

$$
\begin{aligned}
& \text { s.t. } \sum_{\left\{j \mid\left(i_{m}, j\right) \in E\right\}} x_{i_{m} j}(m)-\sum_{\left.\left\{j \mid j, i_{m}\right) \in E\right\}} x_{j i_{m}}(m)=r_{m} \\
& \sum_{\left\{i \mid\left(j_{m}, i\right) \in E\right\}} x_{j_{m} i}(m)-\sum_{\left\{i \mid\left(i, j_{m}\right) \in E\right\}} x_{i j_{m}}(m)=-r_{m} \\
& \sum_{\left\{\begin{array}{c}
j(i, j) \in E, \\
i \neq i_{m}, j_{m}
\end{array}\right\}} x_{\mathrm{ij}}(m)-\sum_{\substack{j \mid(j, i) \in E,\} \\
i \neq i_{m}, j_{m}}} x_{j i}(m)=0 \\
& \sum_{1 \leq m \leq M} x_{\mathrm{ij}}(m) \leq \theta u_{i j} \quad(i, j) \in E \\
& x_{\mathrm{ij}}(m) \geq 0 \quad(i, j) \in E, m=1, \ldots, M
\end{aligned}
$$

In (1), the first, second and third constraint conditions are the flow conservation constraint; the forth constraint condition means that all traffic in a link can't larger than the maximum link capacity. Because of the theory of convex programming [9], the primal problem has the unique optimal solution, and so does the daul problem.

\section{B. The dual problem}

When there is large-scale network, the primal problem has a very high computational complexity. So we use Lagrange function to transform the primal problem into dual problem [10].

First, we define the dual variables $p$ and $q$ as Lagrange Multiplier, which are correspond to the primal constraint conditions. Then we construct the Lagrangian function (2). Note that the ranges of dual variables can be determined according to the theorem of complementary slackness [10].

$$
\begin{gathered}
L(x, p, q)=\theta+ \\
\sum_{m=1}^{M} p_{i_{m}}(m)\left(r_{m}-\sum_{j} x_{i_{m} j}(m)+\sum_{j} x_{j i_{m}}(m)\right) \\
+\sum_{m=1}^{M} p_{j_{m}}(m)\left(-r_{m}-\sum_{i} x_{j_{m} i}(m)+\sum_{i} x_{i j_{m}}(m)\right)
\end{gathered}
$$




$$
\begin{gathered}
+\sum_{m=1}^{M} \sum_{i \neq i_{m}, j_{m}} p_{i}(m)\left(-\sum_{j} x_{\mathrm{ij}}(m)-\sum_{j} x_{j i}(m)\right) \\
+\sum_{(i, j)} q_{i j}\left(\theta u_{i j}-\sum_{m=1}^{M} x_{i j}(m)\right) \\
p \text { free; } q \leq 0 ;
\end{gathered}
$$

Equation (2) could be simplified into (3):

$$
\begin{gathered}
L(x, p, q)=\sum_{m=1}^{M} r_{m}\left(p_{i_{m}}(m)-p_{j_{m}}(m)\right) \\
+\left(1+\sum_{(i, j)} u_{i j} q_{i j}\right) \theta+\sum_{m=1}^{M} \sum_{(i, j)}\left(p_{j}(m)-p_{i}(m)-q_{i j}\right) x_{i j} \\
p \text { free; } q \leq 0 ;
\end{gathered}
$$

Therefore, according the theory of convex programming [9], we can get the daul problem:

$$
\begin{gathered}
\quad \max \sum_{m=1}^{M} r_{m}\left(p_{i_{m}}(m)-p_{j_{m}}(m)\right) \\
\text { s.t. } \quad \sum_{(i, j)} u_{i j} w_{i j}=1 \\
p_{j}(m)-p_{i}(m)+\sum_{(i, j)} w_{i j} \geq 0 \forall m, \forall(i, j) \\
w_{i j} \geq 0 \quad \forall(i, j)
\end{gathered}
$$

Where $w_{i j}$ donates the weight of link, $w_{i j}=-q_{i j}$.

According to the theorem of complementary slackness, when the primal optimal solution is greater than zero, the corresponding dual constraint condition must equal to zero:

$$
\begin{gathered}
p_{j}(m)-p_{i}(m)+\sum_{(i, j)} w_{i j}=0 \\
\sum_{(i, j)} w_{i j}=p_{i}(m)-p_{j}(m)
\end{gathered}
$$

And when the primal optimal solution isn't greater than zero, the corresponding dual constraint condition must be greater than zero:

$$
\begin{gathered}
p_{j}(m)-p_{i}(m)+\sum_{(i, j)} w_{i j} \geq 0 \\
\sum_{(i, j)} w_{i j} \geq p_{i}(m)-p_{j}(m)
\end{gathered}
$$

According to (6) and (8), we can analysis that: when the primal and dual problem obtain the optimum values, the selected path has the lowest weight sum.

\section{TRAFFIC MATRIX BASED LOAD BALANCING ROUTING SCHEME}

Through the above analysis, we concluded that: the primal optimal object to minimize the maximum link utilization, can be transform to the dual optimal object function:

$$
\max \sum_{m=1}^{M} r_{m}\left(p_{i_{m}}(m)-p_{j_{m}}(m)\right)
$$

Where $r_{m}$ donates the traffic demand from router $i$ to router $j$. And $p_{i m}(m)-p_{j m}(m)$ donates the weight sum of a path from router $i$ to router $j$. The dual optimal object could be construed as: the OD-pair with a larger traffic demand should be assigned a path with minimum weight.

For this reason, we propose a traffic matrix based load balancing routing scheme, the pseudocode is as follows:

//Initialize $\boldsymbol{T M}$ and all the road capacity $u_{l}$;

// Initialize $f_{l}=0 ; w_{l}=1$;

1) $\mathbf{T M}=\left\{r_{l}, r_{2}, \ldots, r_{M} \mid r_{i j} \neq 0, i \in \boldsymbol{V}, j \in \boldsymbol{V}\right\}$

2) $\boldsymbol{U}=\left\{\left(u_{1}, f_{l}\right), \ldots,\left(u_{L}, f_{l}\right) \mid u_{l}>0\right\}$

3) $\boldsymbol{W}=\left\{w_{1}, w_{2}, \ldots, w_{L} \mid w_{l}=1\right\}$

4) $\boldsymbol{G}=G(\boldsymbol{V}, \boldsymbol{E}, \boldsymbol{W})$

// Set a threshold K.

//Prioritise pathes (with minimum weight) to maximum $r_{m}$ (above K).

5)for $\left(r_{m} \geqq \mathrm{~K}, r_{m} \in\{\boldsymbol{T M}\}\right) \quad\{$

6) $r_{\max }=\max \{\boldsymbol{T M}\}, \quad\left(r_{\max }=r_{s d}\right)$

//Remove the link whose capacity less than $r_{m}$.

7)if $u_{l}<r_{\max }, w_{l}=\infty,(l=1, \ldots, L)$

//Based on the current link weights, perform the shortest path algorithm.

8) $\boldsymbol{G}=\boldsymbol{G}(\boldsymbol{V}, \boldsymbol{E}, \boldsymbol{W})$,

Path $\left\{r_{\text {max }}\right\}=\operatorname{OSPF}\{\mathrm{i}, \mathrm{j}\}$,

//Remove the corresponding bandwidth for $r_{\max }$, change the $f_{l}$, while the $f_{l}$ can be change only once. Improve the link weights, which the $r_{\max }$ pass through.

9)for all $l \in E$,

$$
\begin{aligned}
& \text { if } l \in \text { Path }\left\{r_{\text {max }}\right\} \\
& \text { if }\left(f_{l}=0\right)\left\{u_{l}=u_{l^{-}} r_{\text {max }}, f_{l}++\right\} \\
& w_{l}=w_{l}+1,
\end{aligned}
$$

//Remove $r_{\text {max }}$

10) $\mathrm{TM}=\{\mathrm{TM}\}-\left\{r_{\max }\right\}$

//For elements less than K, just perform the shortest path algorithm, without performing step 9).

11)else perform step 8).

\section{EXPERIMENTAL ANALYSIS}

We use OMNET++ to run the experiment simulation based on NSFnet topology. The topology consists of $1 \mathrm{RM}, 14$ routers and 42 links, each link bandwidth is $100 \mathrm{Mbps}$. Every router sends random flows to any other routers, while the duration of flows follow the random distribution between 10 Mbps to $70 \mathrm{Mbps}$. The transmission time interval follow the random distribution between $10 \mathrm{~ms}$ to $30 \mathrm{~ms}$.

When we perform the traffic matrix based load balancing routing scheme, RM updates the local traffic matrix every 15 seconds, and the threshold is set to $60 \mathrm{Mbps}$. 
The comparation of the average bandwidth utilization within 60 minutes in SINET and the traditional OSPF is shown in Fig. 2.

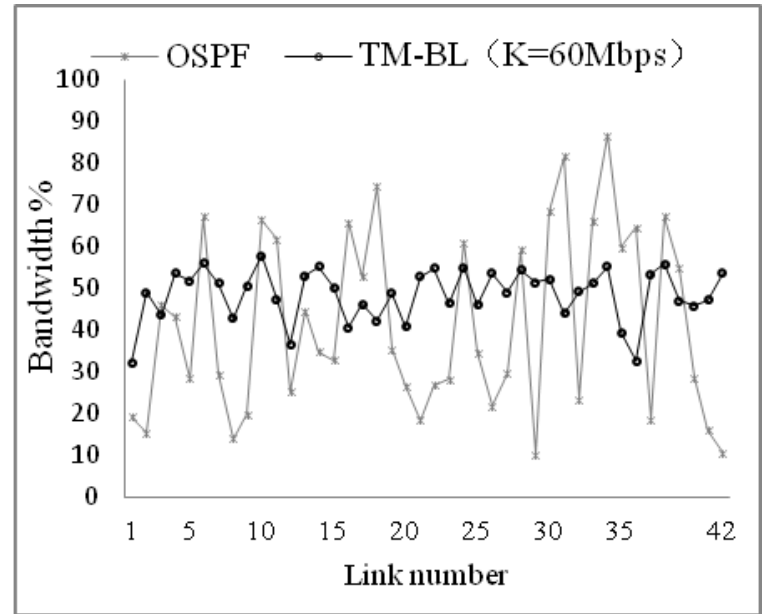

Fig.2 The link bandwidth utilization

As shown in Fig.2, when performing OSPF scheme, the lowest link bandwidth utilization are 10\%, and the highest link bandwidth utilization are $86 \%$. The link bandwidth utilization variance is about $23 \%$. While in the traffic matrix based load balancing scheme, the lowest and highest traffic load are about $32 \%$ and $57 \%$ respectively. The link bandwidth utilization variance is about $6 \%$. The link bandwidth utilization variance reflects the degree of load balance.

\section{CONCLUSION}

The estimation of traffic matrix in SINET adopts a distributed computing mode, and can be achieved at line speed. We use traffic matrix as the constraint, model on load balancing routing problem, and transform the primal problem into dual problem. Then a traffic matrix based load balancing routing scheme is proposed to achieve the optimization goal of the dual problem. The experiment result shows that, compared with traditional routing scheme, the traffic matrix based load balancing routing could reduce congestion effectively and realize load balancing.

\section{Acknowledgment}

This work was supported by the National Basic Research Program of China (973) (2013CB329100); The National Natural Science Foundation of China (61232017, 61271200).

\section{References}

[1] Z. Hongke, L. Hongbin. "Fundamental Research on Theories of Smart and Cooperative Networks". Acta Electronic Sinica, 2013,41(7): 12491254.

[2] L. Hongbin, C. Zhe, Z. Hongke, et al. "CoLoR: an Information -Centric Internet architecture for innovation". IEEE Network Magazine, 2014 28(3):4-10.
[3] L. Hongbin, C. Zhe, Z. Hongke, et al. "An approach for efficient, accurate, and timely estimation of traffic matrices". Proceedings of the INFOCOM WKSHPS 2014. Toronto, Canada, April 2014.

[4] Z. Hongke, Q. Wei, C. Hanjie, et al. "Smart Identifier Network: A Collaborative Architecture for the Future Internet". IEEE Network, under review, May 2015.

[5] A. Medina, N. Taft, K. Salamatian, et al. "Traffic matrix estimation Existing techniques and new directions". ACM SIGCOMM Computer Communication Review. ACM, 2002, 32(4): 161-174.

[6] F. Song, R. Li and H. Zhou, Feasibility and Issues for Establishing Network-based Carpooling Scheme. Pervasive and Mobile Computing (2015).

[7] Y. Yang, Z. Jingjing, Y. Jiahai, et al. "Traffic matrix estimation algorithm based on square root filtering". Computer Science, 2009, 36(7):42-45.

[8] N. McKeown, T. Anderson, H. Balakrishnan, et al. "OpenFlow: enabling innovation in campus networks". ACM SIGCOMM Computer Communication Review, 2008, 38(2): 69-74.

[9] A. Tootoonchian, M. Ghobadi, Y.Ganjali "OpenTM: traffic matrix estimator for OpenFlow networks". Passive and active measurement. Springer Berlin Heidelberg, 2010: 201-210.

[10] C. Baolin. "Theory and Algorithms of Optimization". Beijing: Tsinghua University press, 2005. 